\title{
The Massive stellar Population at the Galactic Center
}

\author{
Francisco Najarro ${ }^{1}$, Diego de la Fuente $^{1}$, Tom R. Geballe ${ }^{2}$, \\ Don F. Figer ${ }^{3}$ and D. John Hillier ${ }^{4}$ \\ ${ }^{1}$ Centro de Astrobiología (CSIC/INTA), ctra. de Ajalvir km. 4, 28850 Torrejón de Ardoz, \\ Madrid, Spain ${ }^{2}$ Gemini Observatory, 670 N. A'ohoku Place, Hilo, HI 96720, USA \\ ${ }^{3}$ Center for Detectors, Rochester Institute of Technology, 54 Lomb Memorial Drive, Rochester, \\ NY 14623, USA \\ ${ }^{4}$ Department of Physics and Astronomy, University of Pittsburgh, 3941 O'Hara Street, \\ Pittsburgh, PA 15260
}

\begin{abstract}
We present results from our ongoing infrared spectroscopic studies of the massive stellar content at the Center of the Milky Way. This region hosts a large number of apparently isolated massive stars as well as three of the most massive resolved young clusters in the Local Group. Our survey seeks to infer the presence of a possible top-heavy recent star formation history and to test massive star formation channels : clusters vs isolation.
\end{abstract}

Keywords. stars: early-type -stars: mass loss - stars: winds - stars: abundances - Galaxy: center - infrared: stars

\section{Massive stars and massive star formation in the GC.}

Hosting three of the most massive young clusters in the Local Group (Central, Arches and Quintuplet), the Galactic Center (GC) constitutes an ideal test-bed to investigate massive stars and massive star formation. Whether the latter occurs in a similar way as in the giant molecular clouds elsewhere in the Galaxy, despite the harsh environment pervaded with intense radiation fields and high densities, has been a matter of debate over the last two decades. Several studies have aimed at establishing whether the Initial Mass Functions (IMFs) and Present Day Mass functions (PDMFs) of these clusters are topHeavy and therefore characterized by a shallower slope caused by an excess of massive stars when compared to a Salpeter distribution (Figer et al. 1999; Stolte et al. 2002; Kim et al. 2006; Espinoza et al. 2009; Bartko et al. 2010; $\mathrm{Hu} \beta$ mann et al. 2012). Being the youngest of the three GC clusters, the Arches constitutes an unique laboratory to investigate the shape of the IMF and PDMF. However, the results obtained range from quite shallow slopes (Figer et al. 1999; Stolte et al. 2002; Kim et al. 2006) to Salpeter-like (Habibi et al. 2013) or half-way (Espinoza et al. 2009) and are strongly dependent on the extinction and transformations of the photometric systems.

Over the last decade, new ingredients have been added to the overall picture, as several studies have revealed the presence of a large number of isolated massive stars (Mauerhan et al. 2010a,b) at the GC which is comparable to the massive star population of each of the clusters (Figer et al. 1999, 2002). Such detection of apparently isolated massive stars in this region has raised a further fundamental issue - whether these "massive field stars" are results of tidal interactions among clusters, are escapees from a disrupted cluster, or represent a new mode of massive star formation in isolation (Dong et al. 2015). Following the numerical dynamical simulations from Harfst et al. (2010), and including the effects of stellar evolution and the orbit of the Arches cluster in the Galactic Center potential, Habibi et al. (2014) investigated the first option and found that models were able to 

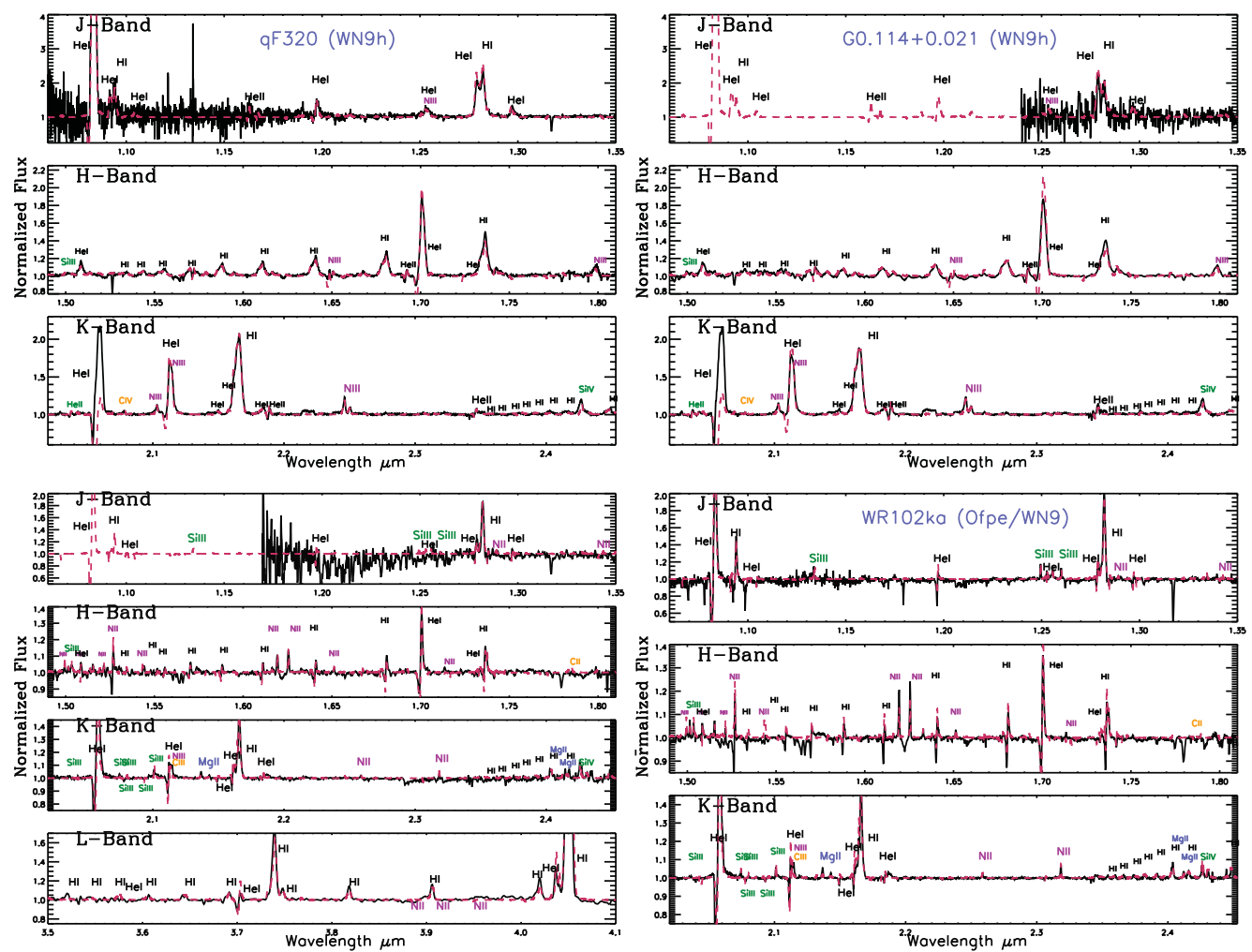

Figure 1. Top. A consistent $\alpha$-element enhancement is found for the Quintuplet sources (left) and isolated massive stars (right). Bottom Breaking the $T_{\text {eff }}$ degeneracy in OIfpe/WN9 stars. Bottom-feft) Q8 in the Quintuplet Cluster. Bottom-right) The isolated WR102ka star (see text).

account for $\sim 60 \%$ of the isolated sources within the central $100 \mathrm{pc}$ as sources drifted away from the center of the clusters. On the other hand, radial velocity measurements of a sample of eight objects in the vicinity of the Arches cluster (Dong et al. 2015) suggest that two of them could have been associated with the cluster while other two likely formed in isolation. The latter option was also inferred for the very luminous WR102ka star (Oskinova et al. 2013) from radial velocity studies and a deep integral-field K-band spectroscopic survey of its surroundings. We note, however, that radial velocity estimates of these objects (mainly OIf + and WNh) may be subject to high uncertainties, as the spectral lines utilized in these studies are severely contaminated by the stellar winds (see below). Thus, further detailed evidence for or against these scenarios is still lacking and awaits precise proper motion measurements (currently underway) providing 3-D velocities of the sources relative to the clusters.

Confronting the stellar properties of the isolated sources, including ages and abundances with those of the putative feeding clusters (Quintuplet and Arches) through detailed spectroscopic studies, may constitute a major step to differentiate among the above scenarios. Comparison of the results of the quantitative model-atmosphere analysis to theoretical isochrones will allow us to determine if these stars were born in single co-eval cold molecular cloud event or formed over an extended (eg, 1-10Myr) period. Obtaining metal abundances from these "field" objects is crucial to understand the metal 

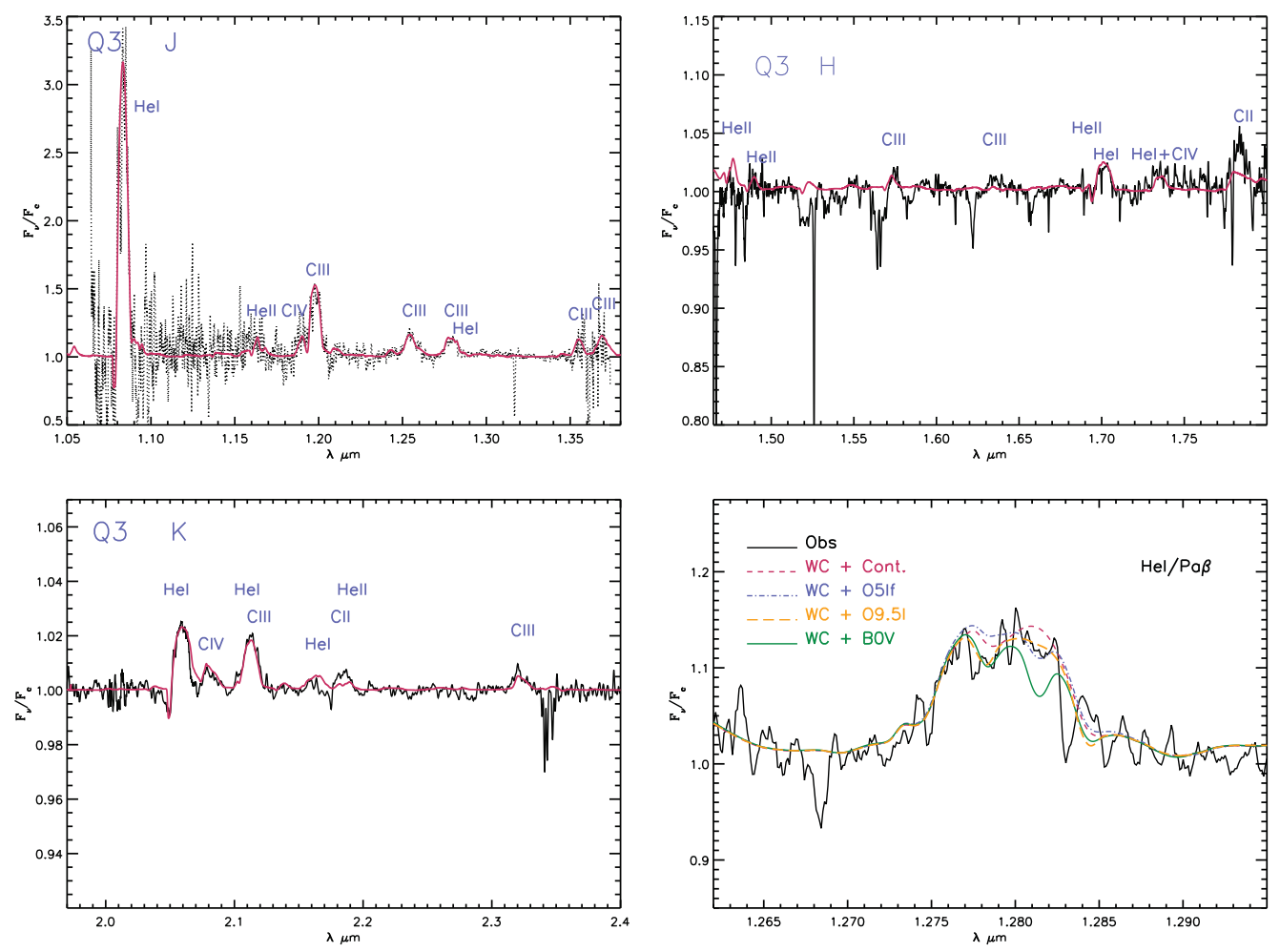

Figure 2. First detection of stellar lines in the NIR spectra of the dusty WCL proper members of the Quintuplet Cluster. J,H and K band observations and model fits for GCS4 (Q3). The bottom-right plot displays the potential of $\operatorname{Paschen} \beta$ to trace the presence of the OB companion.

enrichment history of the GC and to test whether these isolated stars have followed a metal-enrichment scenario different than those in the GC clusters.

The Quintuplet and Arches clusters provide the stellar reference sources to perform such studies. At its present evolutionary phase (age 4 Myr, Figer et al. 1999) the massive members of the Quintuplet Cluster are currently WN9-10h (plus a WN6), weak lined WC9, OIf+ stars and LBVs (Figer et al. 1999; Liermann et al. 2009), while the massive population of the younger Arches cluster is dominated by WN8-9h and OIf + stars (Figer et al. 2002; Najarro et al. 2004; Martins et al. 2008). These spectral types basically encompass all the the isolated evolved massive stars identified from recent follow-up spectroscopic observations of Pa $\alpha$ emission objects in the GC (Mauerhan et al. 2010a,b) which show quite similar spectral morphology to those present in the Quintuplet and some of the brightest Arches members.

Abundance analyses may as well help to distinguish between top-heavy and standard star formation in the region. In a top-heavy IMF scenario, the occurrence of a larger number of type II supernovae produce enhanced yields of $\alpha$-elements, resulting in an increase of $\alpha$-element vs Fe (the main suppliers of iron are type Ia SNe). Najarro et al. (2004, 2009) have shown that quantitative NIR spectroscopy of high-mass stars may provide estimates of both absolute abundances and abundance ratios, telling us about the global integrated enrichment history up to the present, placing constraints on models of galactic chemical evolution, and acting as clocks by which chemical evolution can be measured. 


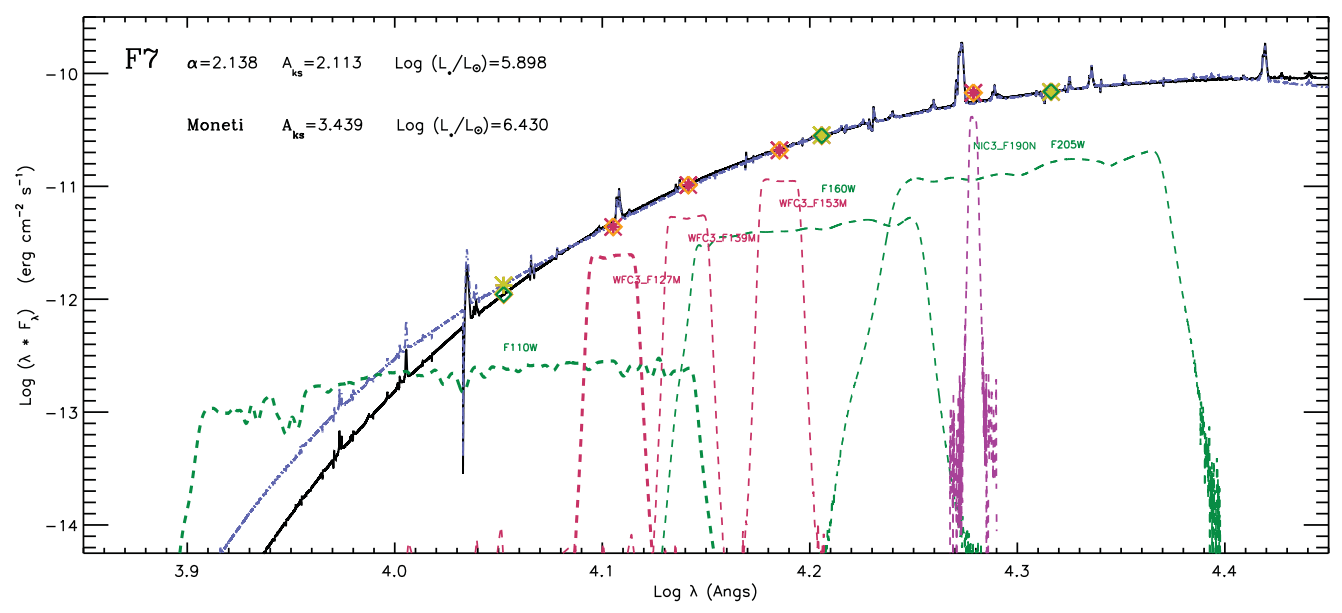

Figure 3. The importance of the extinction law to infer Luminosities and masses (see text).

\section{Observations and results}

Over the last five years we have been carrying an observing program at GEMINI North to obtain high $\mathrm{S} / \mathrm{N}$, medium-resolution spectra of the most massive stars in the Quintuplet (including the dusty WCL proper members) as well as isolated massive stars in the inner GC. So far, around 20 massive stars have been observed. We are currently modeling the early-type spectra with the CMFGEN code (Hillier \& Miller 1998) to obtain physical and chemical properties and present below some results from our ongoing analysis:

We obtain a clear $\alpha$-element enrichment from the analysis of the Quintuplet WNh stars, consistent with the results derived for the LBVs (Najarro et al. 2009) which denoted a clearly enhanced $\alpha / \mathrm{Fe}=2$ ratio with respect to solar (Fig.1-right).

Stellar abundances of the isolated objects (Fig.1-left) seem to show a similar trend on average, with the presence of even higher $\alpha$-element enrichment in some cases.

Our new high $\mathrm{S} / \mathrm{H}$ spectroscopic $\mathrm{J}, \mathrm{H}$ and $\mathrm{K}$ data provide important diagnostic lines (N II-III, SirI-IV, CiI-IV, etc), which are crucial not only for abundance determinations but also to constrain stellar properties. As an example, previously found uncertainties in $T_{\text {eff }}( \pm 6000 \mathrm{~K})$ for the Ofpe/WN9 objects at the GC (Najarro et al. 1997; Martins et al. $2007)$ due to the lack of He II lines in the spectra, are drastically reduced $( \pm 1000 \mathrm{~K})$ by making use of the N II/N III and Si III/Si IV ionization equilibria. This is shown in Fig. 1bottom, where model fits are displayed for two OIfpe/WN9 stars in our sample (Q8 in the Quintuplet cluster and WR102ka, an object relatively far from the three clusters).

When available the $\mathrm{HI}$ and $\mathrm{He}$ lines at $\mathrm{P} \beta$, provide an excellent $\mathrm{He} / \mathrm{H}$ ratio diagnostic, allowing much more accurate He abundance determinations than those performed by means of K-Band spectra. Simple blue (He I) to red (H I) peak ratios may be used (see $\mathrm{P} \beta$ complex all four J-Band spectra in Fig. 1)

Radial velocity estimates, if obtained for OIf + and WNh stars, require detailed modeling of the observed spectra (Figer et al. 2004). For the OIf + stars, the He II absorption lines, which are decent diagnostics for OV and for some OI stars with weak-to-moderate stellar winds start to be filled by the stellar wind, producing an effective blue-shift as high as $80-90 \mathrm{~km} \mathrm{~s}^{-1}$. This may have important consequences when associating the radial velocities of these objects with the nearby gas and clusters. Further, even quantitative modeling may suffer from high uncertainties. Our fits to WR102ka making use of the full J, H and $\mathrm{K}$ band spectra (Fig. 1-bottom-right) reveal a radial velocity of $\sim 100 \mathrm{~km} \mathrm{~s}^{-1}$. 
This value differs significantly from the $60 \mathrm{~km} \mathrm{~s}^{-1}$ obtained by Oskinova et al. (2013) by means of only K-band spectra and with a slightly (\%25) lower spectral resolution.

We detect, for the first time, the WR stellar lines in the NIR SEDs of the dusty WCL proper members in the Quintuplet Cluster (see Fig.2). The deep J band spectra, where the dust contribution weakens clearly display the presence of the He I-II and C III-IV lines of the WC9 component. Further, the huge S/N achieved in the $\mathrm{H}$ and $\mathrm{K}$ band allows to clearly identify and model the stellar lines which are severely diluted by the dust continuum. Thus, we confirm spectroscopically the WR+OB binary nature of these systems and, fitting spectrophotometric data, derive the individual contributions of the dust, WR and OB components to the total SED of the system. Figure 2 displays as well the potential of the observed $\mathrm{P} \beta$ line profile to infer the nature of the OB-component.

Finally, it is worth mentioning the crucial role of extinction, a skeleton on everyone's closet, and its implications on the inferred luminosities and masses of the massive stars and therefore on the IMFs (PDMFs) of the clusters. Figure 3 displays two different reddening approaches to the SED of the stellar model which best reproduces the K-band spectrum of the WNh star F7 in the Arches cluster. Both extinction laws match satisfactorily the observed HST photometry from J to K band but yield Luminosities with differ by more than 0.5 dex. A much lower luminosity and stellar mass (70 vs $\left.130 M_{\odot}\right)$ is obtained if a simple $\mathrm{A}_{\lambda} \propto\left(\lambda / \lambda_{K}\right)^{\alpha}$ relation is used for the whole NIR compared to previously utilized (Figer et al. 2002; Najarro et al. 2004) laws. By securing spectra of the O dwarfs in the Arches, for which the scatter in intrinsic K-Band luminosity is much lower than for the WNh stars, one could definitely discern between both extinction approaches.

F.N. and D.dF. acknowledge grants FIS2012-39162-C06-01, ESP2013-47809-C3-1-R and ESP2015-65597-C4-1-R

\section{References}

Bartko, H., Martins, F., Trippe, S., et al. 2010, ApJ, 708, 834

Dong, H., Mauerhan, J., Morris, M. R., Wang, Q. D., \& Cotera, A. 2015, MNRAS, 446, 842

Espinoza, P., Selman, F. J., \& Melnick, J. 2009, A\& $A$, 501, 563

Figer, D. F., McLean, I. S., \& Morris, M. 1999, ApJ, 514, 202

Figer, D. F., Najarro, F., Gilmore, D., et al. 2002, ApJ, 581, 258

Figer, D. F., Najarro, F., \& Kudritzki, R. P. 2004, ApJ (Letters), 610, L109

Habibi, M., Stolte, A., Brandner, W., Hußmann, B., \& Motohara, K. 2013, A\&sA, 556, A26

Habibi, M., Stolte, A., \& Harfst, S. 2014, A\& A, 566, A6

Harfst, S., Portegies Zwart, S., \& Stolte, A. 2010, MNRAS, 409, 628

Hillier, D. J. \& Miller, D. L. 1998, ApJ, 496, 407

Hußmann, B., Stolte, A., Brandner, W., Gennaro, M., \& Liermann, A. 2012, A\&A, 540, A57

Kim, S. S., Figer, D. F., Kudritzki, R. P., \& Najarro, F. 2006, ApJ (Letters), 653, L113

Liermann, A., Hamann, W.-R., \& Oskinova, L. M. 2009, A\&SA, 494, 1137

Martins, F., Genzel, R., Hillier, D. J., et al. 2007, $A \& A$, 468, 233

Martins, F., Hillier, D. J., Paumard, T., et al. 2008, Aछ A, 478, 219

Mauerhan, J. C., Cotera, A., Dong, H., et al. 2010a, ApJ, 725, 188-199

Mauerhan, J. C., Muno, M. P., Morris, M. R., Stolovy, S. R., \& Cotera, A. 2010b, ApJ, 710, 706

Najarro, F., Krabbe, A., Genzel, R., et al. 1997, A\&A, 325, 700

Najarro, F., Figer, D. F., Hillier, D. J., \& Kudritzki, R. P. 2004, ApJ (Letters), 611, L105

Najarro, F., Figer, D. F., Hillier, D. J., Geballe, T. R., \& Kudritzki, R. P. 2009, ApJ, 691, 1816

Oskinova, L. M., Steinke, M., Hamann, W.-R., et al. 2013, MNRAS, 436, 3357

Stolte, A., Grebel, E. K., Brandner, W., \& Figer, D. F. 2002, A\&3A, 394, 459 